\title{
Analyses of MAC Performance for Multi-Carrier based Wireless Sensor Networks
}

\author{
Fei Qin \\ John E. Mitchell \\ Department of Electronic and Electrical Engineering \\ University College London \\ London, UK \\ \{f.qin, j.mitchell\}@ee.ucl.ac.uk
}

\begin{abstract}
In this work we adopt an OFDMA like Multi-Carrier scheme for wireless sensor networks with the aim of improving the performance in the presence of intensive traffic load. To examine the MAC performance, a simple, yet accurate analytic model has been provided, validated by simulation results. We demonstrate that the proposed scheme can achieve better throughput under heavy offered traffic load, as a result of the higher bandwidth utilisation. In addition, energy efficiency and service delay performance can be significantly increased.
\end{abstract}

Keywords- Multi-Carrier, OFDMA, Wireless Sensor Networks, Throughput, Service Delay, Energy Efficiency

\section{INTRODUCTION}

Wireless Sensor Networks (WSNs) have been widely promoted over the last decade, where they were designed to monitor various environmental parameters, e.g. temperature, light, and humidity. Recent applications of WSNs have expanded into more advanced fields, for example structural health monitoring, manufacturing automation monitoring, and multimedia surveillance etc. In these advanced applications, WSNs could be re-recognized as a data intensive network instead of the traditional low duty cycle network. When exposed with the heavy offered traffic load, the collision probability in the wireless channel is increasing rapidly, which decreases the successful transmission ratio and delivered throughput. Consequently, the energy consumption of the WSN device will increase as well, due to the wasted energy on failed transmission attempts. Furthermore, WSNs are usually multihop networks, with the competition for bandwidth caused by hidden terminals and the exposed terminals further degrading the network performance in the terms of throughput decrease and energy consumption increase. However, the flexibility of a multi-hop network is the main motivation for sensor networks, e.g. in the Golden Gate Bridge Monitoring Project [1], where 52 MicaZ devices have been deployed forming a multi-hop network. To avoid the problems mentioned above, the authors set all nodes to sample at the same time but transmit data back one after another, which means that there is only one transmitter in the whole network at one time slot to avoid the potential competition. All these problems can have negative effects on the throughput, and the energy efficiency of the network, that limit the application of sensor networks in advanced, traffic intensive fields.

Attempting to solve these problems, some researchers [2;3] have proposed using Code Division Multiple Access (CDMA) based wireless technology to increase MAC layer efficiency, and as a result, increase the performance of WSN. Due to the well designed orthogonal code set, CDMA is able to increase the channel multiplexing. However, a CDMA based network will be highly dependent on accurate power control to level the received power of all incoming signals to avoid interference. Similar requirements exist in the time synchronization, since the orthogonality will rapidly decrease due to the shift of code words. The costs of these requirements are acceptable in a star topology network (e.g. cellular system), but may be overly complex for a multi-hop network. Another proposed approach is the multi-radio based system [4;5]. In this architecture, high flexibility can be expected, due to the use of independent and simultaneously accessible radio transceivers. Since each transceiver can operate in different channels, simultaneous wireless communication with multiple devices will be enabled in the MAC layer. In turn, this will provide the performance gain in the terms of minimizing service delays, and increasing network throughput. However, as expected, the performance gain obtained in this approach is at the expense of additional radio transceivers, which will increase both the power consumption and the system cost.

Orthogonal Frequency Division Multiple Access (OFDMA) has been widely employed in cellular and data networks (e.g. LTE and WiMax system), because of its high flexibility in allocating spectrum to multiple users and its robustness in the presence of multi-path effects. The basic concept of OFDMA system is to divide the wireless channel into several subcarriers, sets of which can be allocated to different devices to achieve frequency multiplexing. Therefore, an OFDMA system can achieve similar flexibility as the multi-radio system but with a single transceiver. Thus, we are motivated to exploit these advantages of OFDMA as an efficient multiplexing approach for wireless sensor networks. However, some wellknown characteristics of OFDMA, in particular the sensitivity to Carrier Frequency Offset (CFO) and the high Peak to Average Power Ratio (PAPR) pose challenges to its application in low cost, multi-hop sensor network. Due to these characteristics, OFDMA is only used in the downlink mode by the LTE and WiMax systems, where there is only one sender and several receivers. If OFDMA were to be used in WSNs, where there will be several senders, the carrier frequency offset caused by frequency variations between different sender's Local Oscillator (LO) frequency variation would cause significant Inter Channel Interference (ICI) and Multi User Interference (MUI) [6], which would dramatically degrade the 
system error performance. In addition, the high PAPR can cause low power efficiency and increase device cost, since it requires highly linear power amplifiers to avoid signal distortion. As such problems have been significant existed network, we suggest that in a WSNs consisting of hundreds of nodes and tens of hops, the OFDMA architecture without any modification will be even more challenging to implement.

To avoid these problems but benefit from the advantages (i.e. spectrum flexibility and resistant to multi-path effect) of the OFDMA architecture for sensor networks, we have analytically examined the cause of these problem, and experimentally demonstrated one possible modified MultiCarrier architecture for WSN in [7], where pulse shaping, differential modulation, and chip spreading have been jointly employed to increase the CFO resistance and decrease the PAPR. The proposed physical layer design has been validated by the experimental approach, where the proposed system shows very good performance in against the above mentioned problems.

In this paper we investigate to what degree WSNs can benefit from this OFDMA like multi-carrier based architecture. In $[8 ; 9]$, researchers have argued that the simple increase of spectrum flexibility without an increase in overall bandwidth will not increase the throughput performance of network in the MAC layer. However, as the throughput is not necessarily the priority in WSNs, we consider if the multi-carrier architecture can offer other benefits such as energy efficiency. Due to experiment limitations on the network performance, in this work, we present an analytical approach to demonstrate the performance of this multi-carrier system and show that energy efficiency can be achieved while still maintaining high throughput when exposed with heavy offered traffic load.

This paper is organised as follows: In section II, we present an analysis of the system performance at the MAC layer for a multi-carrier based sensor network, which based on the previous work of [7] forming the physical layer of the system to be investigated. In section III, a simulation based comparison is shown to validate the accuracy of proposed analytic model. In the last section, we summarise our findings.

\section{The ANALYTIC MOdEL OF MUlti-CARRIER BASED MAC PROTOCOL}

In our previous work, one of the possible multi-carrier physical layer architectures has been proposed and examined through an experimental approach, which enables the devices in the wireless sensor network to use the OFDMA like multiplexing function with great flexibility [7]. Differing from traditional devices (e.g. CC2420) that can only switch between independent channels, devices with this new architecture will be able to transmit data over any number of sub-carriers at the same time as well as enabling several devices to transmit concurrently by occupying different sub-carriers. To quantify the potential performance benefits, an analytic model has been developed, in which the overall bandwidth is equal to one channel in the traditional system, e.g. 2MHz in IEEE 802.15.4 as shown in Fig. 1. This restriction normalises the performance gain highlighting the benefit obtained due to channel sharing flexibility without any bandwidth increase.

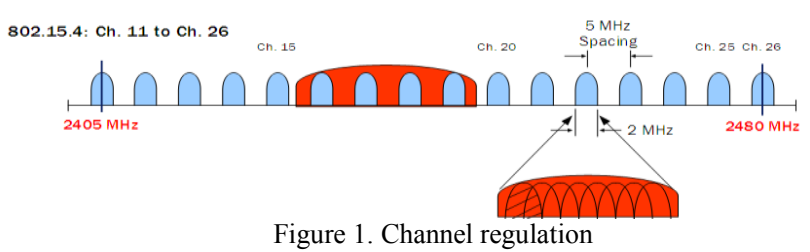

The proposed analytic model is based on the Slotted Aloha, as it is the simplest MAC protocol in a wireless network. Further extension of the proposed analytic model to other MAC protocols, like Carrier Sense Multiple Access (CSMA) or other more complex random access MAC protocols, as well as an unsoltted model is straightforward.

\section{A. 2-Level Renewal Process}

The analytic model was built on the concept of a level based renewal process, which was first proposed to analyze the performance of MAC protocols in [10]. This approach can significantly simplify the mathematical analysis without loss of accuracy.

Fig. 2 shows the renewal cycle scheme of slotted aloha. Each time a device has a packet ready to send, it will backoff a random number of time slots following the specified retransmission policy. After the backoff period, the device will send a packet via the wireless channel. If there is only one device sending, then this attempt will be successful (marked as $\mathrm{X} 2$ cycle in level 1) and the device will receive the ACK packet from the receiver later that time slot, otherwise this transmission attempt will be failed due to a collision (marked as $\mathrm{X} 1$ cycle in level 1). There can be $i(i>0) \mathrm{X} 1$ cycles before one $\mathrm{X} 2$ cycle, where $i$ depends on the successful probability of transmission. In level 2, there will always be a successful transmission event in each $\mathrm{Y}$ cycle, while the average length of $\mathrm{Y}$ cycle depends on the performance of the $\mathrm{X}$ cycle. After the calculation of the Y cycle's average length, the performance factors of the current node, including throughput, service delay and energy efficiency can be derived.

The Multi-Carrier based mode will follow the main principle of slotted aloha. The only modification is that the device will randomly choose a sub-carrier from the set of Bsubcarriers to send the packet. Consequently, the successful transmission condition is determined by whether there are other devices transmitting on the same sub-carrier. Thus, it will decrease the collision probability and increase the overall performance.

A single-cluster wireless sensor network with $N$ devices will be analyzed in this model. To simplify this analysis, all devices are considered to be placed within the transmission range of one another, so there will be no hidden terminals in the network. All the devices are random distributed with uniform probability around the sink device.

\section{B. Analytic Model}

In this paper, three retransmission policies including uniform backoff, binary exponential backoff, and geometric backoff will be analysed. When a packet transmission fails, a retransmission is scheduled after a random backoff period 


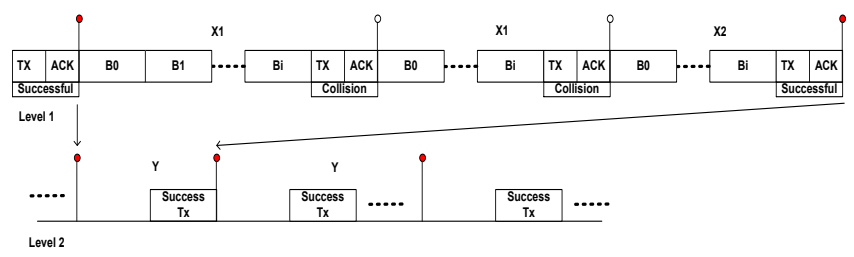

Figure 2. Renewal process

determined by a specific retransmission policy. Let $W_{j}$ be the $j$ th backoff period in units of time slots, then the $i$ th retransmission takes place at the beginning of the $W_{j}$ th available slot after the last failed transmission.

Although we are aware that the duty cycle in WSN is usually very low, each WSN device in this model is assumed to work in saturation conditions. Such an assumption demonstrate the comparative performance of different networks as is likely to occur in the congestion scenario, which is thought to be the most harmful factor to the performance of WSNs system.

\section{Uniform Backoff}

Under a Uniform Backoff (UB) policy, all $W_{j}$ are uniformly distributed in the same range from $[0 \sim w-1]$. Where $w$ is the backoff window size. The current device will start to transmit in the current slot with the probability, defined by

$$
\tau=\frac{1}{\bar{X}}
$$

where $\bar{X}$ is the average length of $X$ cycles:

$$
\begin{aligned}
\bar{X} & =T_{B O}+T_{t x} \\
& =\frac{w-1}{2}+1 \\
& =\frac{w+1}{2}
\end{aligned}
$$

Under the assumption that the channel is ideal, i.e. no transmission error, the conditional probability that a device transmits a packet successfully in single carrier system can be derived as:

$$
P_{s u c}^{s}=(1-\tau)^{N-1}
$$

where $N$ is the number of devices within the network. If none of the other $N-1$ devices are trying to transmit in the same slot, then the current device's transmission will be successful. Hence, $P_{\text {suc }}^{s}$ represents the ratio of X1 cycle number to X2 cycle number in level 1 within a single carrier system, which means that a level 2 cycle contains a average number of $1 / P_{s u c}^{s}$ level 1 cycles with average length of $\bar{X}$. The extension to realistic non-ideal channel assumption (the packet will be transmitted with the error probability instead of perfect transmission) will be discussed in section II.C.

As mentioned in pervious sections, the main performance gain of the multi-carrier system in this model was contributed by increased sharing of the wireless channel, i.e. the increase of $P_{\text {suc }}$ :

$$
P_{\text {suc }}^{m}=\prod_{n=1}^{N-1}\left[(1-\tau)+\tau \cdot \frac{B-1}{B}\right]
$$

Compared with equation (3), the probability of successful transmission is constituted of two parts: the first part is identical to equation (3): the current device will not transmit in the current slot, while the second part can be understand as: the current device will transmit in the current slot, but the chosen sub-carrier from the overall $B$ sub-carriers is different to the one chosen by the current device. Normally $P_{\text {suc }}^{m}$ is much smaller than $P_{s u c}^{S}$, especially when the number of competing nodes is large.

Thus, the average length of a level 2 cycle can be defined as:

$$
\bar{Y}=\frac{\bar{X}}{P_{\text {suc }}^{m}}
$$

The throughput of single device can be simply calculated, as only one packet can be successfully transmitted in each level 2 cycle:

$$
T P_{S}=\frac{L}{\bar{Y}}
$$

where $\mathrm{L}$ is the payload in one slot time.

Therefore, considering that $N$ sensor nodes exist in the network, the network throughput at the sink node can be defined as:

$$
T P=N \cdot T P_{S}
$$

\section{Binary Exponential Backoff}

Considering a Binary Exponential Backoff (BEB) policy, the backoff period is uniformly distributed in a binary, exponentially expanding range. After each unsuccessful transmission, the backoff window size will be doubled. In other words, $W_{j}$ is uniformly distributed in $\left[0 \sim 2^{i-1}(w-1)\right]$, where $i$ is the number of the retransmission, $w$ is the initial backoff window size. Clearly, the length of $\mathrm{X}$ cannot be directly estimated as in the uniform backoff scenario, since it will be dependent on the successful transmission rate. With a given $P_{\text {suc }}$, the average length of the level $1 \mathrm{X}$ cycle can be written as:

$$
\begin{aligned}
& \bar{X}=P_{s u c}\left(T_{B O}^{0}+T_{t x}\right) \\
& +P_{s u c}\left(1-P_{s u c}\right) \frac{\left(T_{B O}^{1}+T_{t x}+T_{B O}^{0}+T_{t x}\right)}{2} \\
& +P_{s u c}\left(1-P_{s u c}\right)^{M-1} \frac{\left(\sum_{m=0}^{M-1} T_{B O}^{m}+T_{t x}\right)}{M} \\
& +\left(1-P_{s u c}\right)^{M} \frac{\left(\sum_{m=0}^{M-1} T_{B O}^{m}+T_{t x}\right)}{M}
\end{aligned}
$$

where $M$ is maximum retry number, after which the current device will discard the packet. $T_{B O}^{i}$ is the expectation of the back off slot, which can be expressed as: 


$$
E\left(T_{B O}^{i}\right)=\frac{2^{i} w-1}{2}
$$

By applying $T_{B O}^{i}$ to equation (7), the expectation of $X$ can be calculated in equation (9):

$$
\begin{aligned}
\bar{X}= & \sum_{m=0}^{M-1} P_{s u c}\left(1-P_{s u c}\right)^{m} \frac{\sum_{i=0}^{m} \frac{2^{i} w-1}{2}+1}{m+1} \\
& +\left(1-P_{\text {suc }}\right)^{M} \frac{\sum_{i=0}^{M-1} \frac{2^{i} w-1}{2}+1}{M}
\end{aligned}
$$

As we expected, the average length of $X$ is highly dependent on the value of $P_{s u c}$. Recall the consistence of $P_{\text {suc }}$ shown in equation (4), we can conclude that $\bar{X}$ and $P_{\text {suc }}$ have formed a non-linear system shown in equation (11).

The equations of (11) represent a non-linear system with two unknowns, $\overline{\mathrm{X}}$ and $\mathrm{P}_{\mathrm{suc}}^{\mathrm{m}}$, both of which are continuous and monotonic functions in the range of $(0,1)$. Both equations represent a non-linear system with two unknown variables, $\bar{X}$ and $P_{\text {suc }}$. With given scenario related parameters, these nonlinear system can be solved numerically, following the method provided in [11].

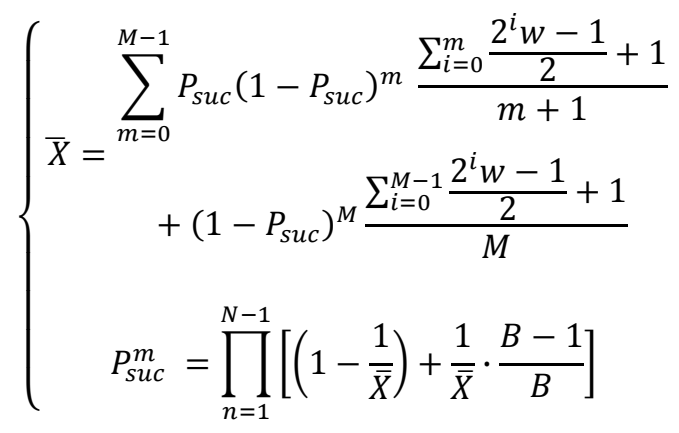

Geometric Backoff

For a Geometric Backoff (GB) policy, the backoff period is geometrically distributed with parameter $q$, where the device will start transmission in the current slot with probability of $q$.

Obviously, the expectation of $T_{B O}$ is only determined by $q$, which can be expressed as $1 / q$. Thus, the average length of the $X$ cycle can be expressed as:

$$
\begin{aligned}
\bar{X} & =T_{B O}+T_{t x} \\
& =\frac{1}{q}+1
\end{aligned}
$$

All the other equations needed for the GB model for both single carrier system and multi-carrier systems are similar to the UB model discussed previously, and can be easily solved following the same approach.

\section{Model Extension}

\section{Non- ideal channel}

A non-ideal channel will cause unsuccessful reception of packets in a real deployment, even without any collision. In the more realistic assumption, equation (7) should be rewritten as:
$\sum_{n=1}^{N} T P_{n}$, since each device will have a different error probability based on their different distances from the sink device.

$$
\begin{gathered}
T P=\sum_{n=1}^{N} T P_{n} \\
=\sum_{n=1}^{N} \frac{L \cdot P_{\text {suc }}^{n}}{\bar{X}} \\
\cong N \cdot \frac{L \cdot P_{\text {suc }} \cdot P R R_{\text {payload }}^{\prime} \cdot P R R_{\text {ack }}^{\prime}}{\bar{X}} \\
=N \cdot T P_{s} \cdot P R R_{\text {payload }}^{\prime} \cdot P R R_{\text {ack }}^{\prime}
\end{gathered}
$$

where $P R R^{n}$ is the Packet Receive Rate (PRR) for device $n$, and is a joint function with the packet length and the distance between device $n$ and the sink device. However, with the assumption that all the devices are randomly placed with a uniform distribution around the sink device, a constant value of PRR expectation can be used for analysis.

\section{Service delay}

Service delay can be defined as the duration from the time that the packet was generated to the end of its successful transmission. The average length of a level 2 cycle represents the time period between two successful transmissions. If $M=\infty$, in other words, a packet will never be discarded, $\bar{Y}$ will be the exact service delay. However, a packet will be discarded after $M$ attempts in a real deployment so that $\bar{Y}$ should be redefined as a combination of the service time for discarded packets and the service time for the current packet.

If we define $P_{d i s}$ as the probability that a packet will be discarded by the current device after $M$ unsuccessful transmissions, $P_{\text {dis }}$ can be expressed as:

$$
P_{\text {dis }}=\left(1-P_{\text {suc }}\right)^{M}
$$

We note that there will be only one successful transmitted packet in every $\frac{1}{P_{d i s}}$ attempted packets.

The average service delay $\bar{D}$ should be the average length of level 2 minus the average length contributed by discarded packets:

$$
\bar{D}=\frac{\bar{X}}{P_{s u c}}\left(1-P_{\text {dis }}\right)
$$

\section{Energy consumption}

Power efficiency in this model is evaluated by the average power consumed for each of the level 2 cycles, i.e., the power consumed for each successfully transmitted packet. As stated previous, each successful transmission is based on several level 1 cycles, which are consisted of three stages: backoff, transmission and receive, with each stage, consuming power with rates: $E_{i}, E_{t}$ and $E_{r}$ respectively. It should be noted that the power consumed in that slot will always be the same whether or not the ACK is successfully received. As a result, the power consumed in this stage has been normalised to a single rate $E_{a}$ to simplify the calculation. The energy consumption for each transmission attempt can be defined as:

$$
\bar{e}=T_{B O} \cdot E_{i}+T_{t x} \cdot E_{a}
$$




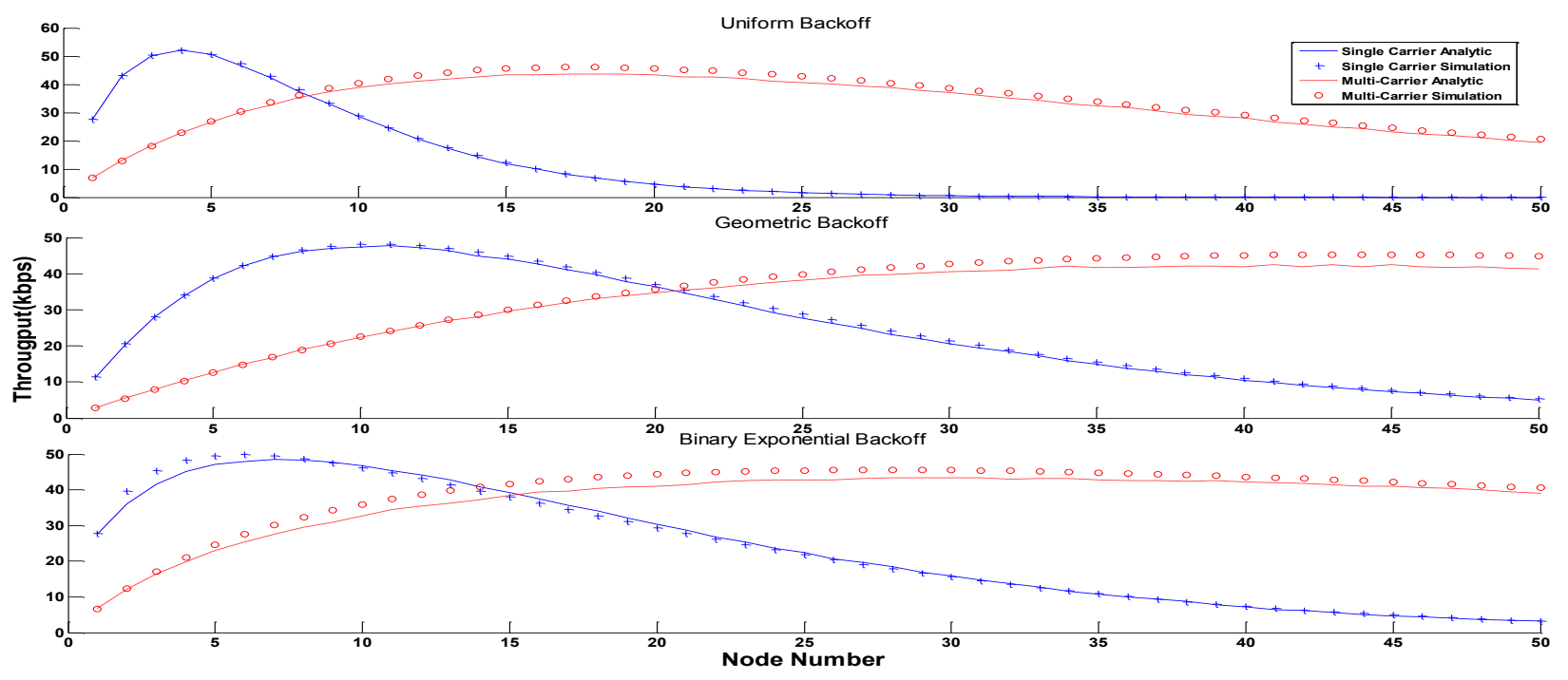

Figure 3. The validation of analytic model using simulation result $(W=8, M=4, q=0.1, B=4)$

where $T_{B O}$ and $T_{t x}$ are related to different scenarios, which can be derived from equation (4), (8), (12).

Considering that current transmission attempt is a success transmission with probability $P_{\text {suc }}$, then the energy consumption for each successfully transmitted packet can be defined as $\bar{E}$ :

$$
\bar{E}=\frac{\bar{e}}{P_{\text {suc }}}=\frac{T_{B O} \cdot E_{i}+T_{t x} \cdot E_{a}}{P_{\text {suc }}}
$$

\section{MODEL VALIDATION AND PERFORMANCE ANALYSIS}

All three retransmission policy based scenarios discussed above have been implemented in the open source network simulator OMNet++ 4.0 and the results are used to validate the accuracy of proposed analytic model

According to the analytic model, all devices are limited to within one hop of the sink device. All the devices are assumed to be uniformly distributed within the Dmax $=40$ meters area. Two RF physical modes have been implemented in the simulation for network performance evaluation: the first mode is the standard IEEE 802.15.4 PHY mode where the RF frontend occupies a bandwidth of $2 \mathrm{MHz}$ and enables a $250 \mathrm{kbps}$ data rate; the second is the proposed multi-carrier system discussed previously, which divides the $2 \mathrm{MHz}$ overall bandwidth into 4 sharable sub-carriers, each of which has a data rate of $62.5 \mathrm{kbps}(250 \mathrm{kbps} / 4)$. We configured that $\Delta f_{C F O}$ follows normal distribution with $\mu=0, \sigma=0.2 d F$ (i.e. $50 \mathrm{kHz}$, according to the $42 \mathrm{kHz}$ frequency offset monitored in an IEEE802.15.4 system through an experimental approach). Therefore the PRR expectation for the analytic model can be derived from experiment data. It worth noting that, as discussed in the previous work of [7], although the proposed multi-carrier system has acceptable performance when the frequency shift is within $\pm \frac{1}{4} \Delta f$, the error expectation of multi-carrier system will still be slightly worse than a single-channel system in the same network scenario. The time slot length was set to be $40 \cdot T s$, (Ts is set to be one symbol duration: $16 \mu \mathrm{sec}$, according to [12]), which equates to 80 bits of data in the packet. The initial backoff windowsize $W$ is set to 8 , with the maximum retransmission attempts $M$ set to 4, and the geometric probability $q$ used in the GB model set to 0.1 . The transmission error performance is derived from the previous experiment result in [7]. Each simulation program takes around 30min, and is repeated 10 times to give an average result.

Throughput is calculated in the sink device and shows how many data packets arrived at the sink device successfully in a given period. The result in Fig. 3 shows a high degree of correlation between the simulation and the proposed analytic model. Therefore, although the throughput of the proposed system is lower than the traditional system because of the lower available traffic load with lower node numbers (since the simulation works in saturation mode, the offered traffic load is only decided by node number), the throughput of the proposed system will be greater than the standard system when the node number crosses a threshold. Furthermore, the throughput of the single-carrier system will trend to zero when the size of network is extremely large, while the proposed system still shows very good performance. The result shows that the proposed system is more suitable for data intensive applications in large scale networks.

Fig. 4 shows the performance of the average service delay for the three different policies. The general trends can be seen in the UB mode: the service delay of single-channel based UB system increases rapidly to the upper bound, while the result from the multi-carrier based UB system increases slowly and converges to the upper bound only when the network size is extremely large. Similar trends also exist in the GB and BEB systems. However, as the average cycle lengths in these two models are larger than the UB model, these trends have been significantly amplified.

In order to calculate the energy performance, we make an approximation of the power consumption of the CC2420 transceiver (as shown in Table 1), which is deduced from the datasheet of the CC2420 [13]. However, the datasheet only 
TABLE I: POWER CONSUMPTION SETTINGS

\begin{tabular}{|c|c|}
\hline & Power Consumption (mA) \\
\hline Tx front-end (PA) & 9 (output 0 dBm) \\
\hline Rx front-end(LNA) & 11.3 \\
\hline Baseband (DAC,ADC) & 4 \\
\hline Digital Processing & 4 \\
\hline Idle (CLK, bus) & 0.4 \\
\hline
\end{tabular}

provides the total power consumption for $\mathrm{Tx}$ and $\mathrm{Rx}$ modes, $17.4 \mathrm{~mA}$ and $19.7 \mathrm{~mA}$ respectively. We make an approximation that the Tx front end consumption is $9 \mathrm{~mA}$ when transmitting at $0 \mathrm{dBm}$, which means that the remaining $8.4 \mathrm{~mA}$ is consumed by other parts. In this case, the $\mathrm{Rx}$ front end power consumption should be $11.3 \mathrm{~mA}$. The data sheet provides an idle state power consumption of $400 \mu \mathrm{A}$, which is treated as consisting of the clock source, $\mathrm{I} 2 \mathrm{C}$ bus communication and other basic functions. The remaining $8 \mathrm{~mA}$ has been assumed to be formed of two parts; $4 \mathrm{~mA}$ for baseband processing, with another $4 \mathrm{~mA}$ for digital processing. Nonetheless, it must be noted these figures are based on assumptions not real test data due to the level of integration and only represent a typical device.

In equation (16), $E_{i}$ is set to $8.4 \mathrm{~mA}$ and $E_{a}$ is set to $18.5 \mathrm{~mA}$. The proposed multi-carrier based WSNs system has been considered with a $1 \mathrm{~mA}$ increase in power consumption in the digital processing component due to the additional signal processing function proposed in [7]. The result, shown in Fig.5, is in the form of energy consumption per cycle, which can be understood as how much energy has been consumed to successfully transmit a packet. The result demonstrates that the proposed multi-carrier system has superior performance over the traditional single-carrier system. For instance, the energy consumption in the single carrier based system increases rapidly with network size is increasing, while the multi-carrier system maintains relatively low power consumption until the network size is as large as 40. This excellent performance in energy efficiency is due to the high availability of the shared wireless channel, i.e. the higher $P_{\text {suc }}$.

The analyses prove that, although the proposed multi-carrier based WSNs may not be able to achieve a significant performance improvement in the throughput of network, i.e. in the form of maximum achieved throughput, the proposed multicarrier scheme does gain in the other important performance metrics, notably, service delay and energy efficiency. The deliverable throughput is also gained when exposed with heavy offered traffic load in dense network.

It should be noted that the proposed system will have access to all the sub-carrier information in Clear Channel Assessment (CCA) operation under a CSMA based MAC protocol, which means the collision probability can be further decreased (i.e. the performance further increased) by avoiding occupied subcarriers. The system can also be designed to work with a wider overall bandwidth, (e.g. the system works across all 16 IEEE802.15.4 channels), which can be expected to give a significant performance improvement compared with the standard IEEE802.15.4 system. Nevertheless, the proposed analytic model may still apply for these scenarios, and the extension should be very straightforward.

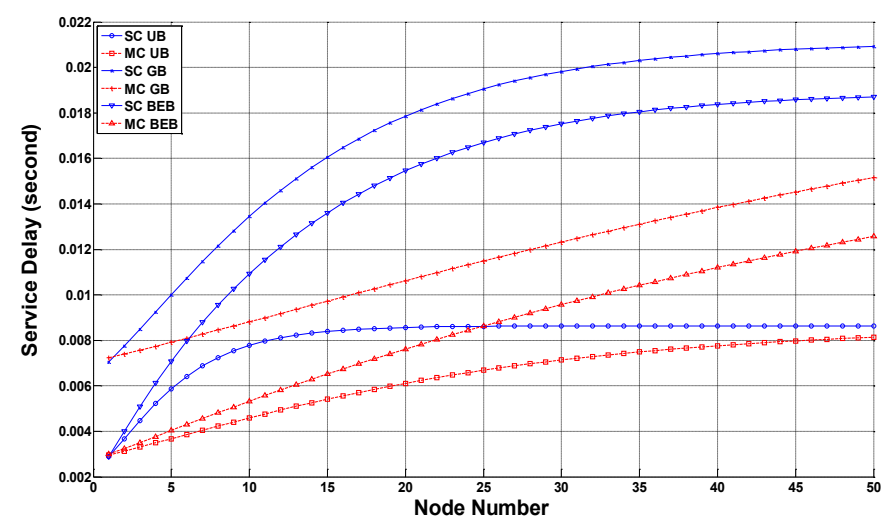

Figure 4. Average service delay for each successfully transmitted packet

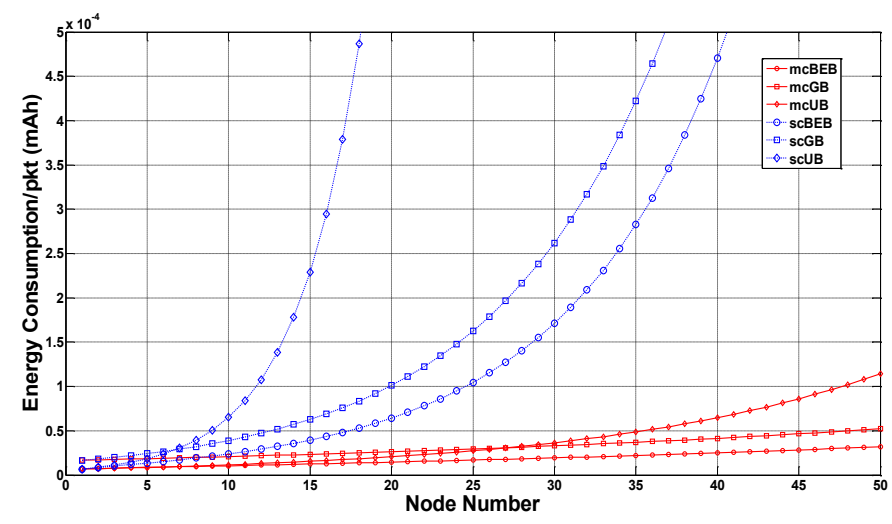

Figure 5. Average energy consumption for each successfully transmitted packet

To simplify the analyses, the analytic model was introduced with the stipulation that each device only occupies one subcarrier. Then it is easy to conclude that, for any device, the performance can be increased, if it occupies more sub-carriers. This could be the best solution to provide different QoS service according to application requirements. However, the assignment of sub-carriers at the network level is expected to be an extremely complex optimization problem, especially in the multi-hop network scenario. This will be studied in our future work.

\section{CONCLUSION}

In this paper, based on a realisable low cost multi-carrier physical layer proposed previously, we demonstrate the potential performance gain that the implementation of this $\mathrm{PHY}$ layer in conjunction with an appropriate MAC layer can achieve. An analytic model has been proposed to evaluate the potential performance gain of proposed architecture under an Aloha based network scenario with multi-carrier modification. A simulation program has been implemented in OMNet ++4.0 to validate this model.

The work proposed has a number of benefits. First, superior energy efficiency can be expected when compared with a single carrier system. This is because of the efficient channel sharing which reduced backoff time and retries time. Second, the proposed multi-carrier base sensor network can have better throughput performance in larger networks with intensive traffic load, due to efficient bandwidth management in dense network scenario. These performance improvements are the 
result of the fact that the transmitting mode is able to transmit over any number of sub-carriers, while receiving mode is able to listen and receive from all sub-carriers. This modification enables the design of MAC protocol with great flexibility and leads to better bandwidth sharing.

\section{ACKNOWLEDGMENT}

This work is jointly supported by a Dorothy Hodgkin Postgraduate Award program (BT \& EPSRC sponsored) and by the Cooperating Objects Network of Excellence (CONET), funded by the European Commission under FP7 with contract numberFP7-2007-2-224053.

\section{REFERENCES}

[1] K. Sukun, S. Pakzad, D. Culler, J. Demmel, G. Fenves, S. Glaser, and M. Turon, "Health Monitoring of Civil Infrastructures Using Wireless Sensor Networks," in 6th International Symposium on Information Processing in Sensor Networks, IPSN 2007, pp. 254-263.

[2] K. Benkic, "Proposed use of a CDMA technique in wireless sensor networks," in 14th International Workshop on Systems, Signals and Image Processing, 2007, pp. 343-348.

[3] B. H. Liu, N. Bulusu, H. Pham, and S. Jha, "CSMAC: A novel DS-CDMA based MAC protocol for wireless sensor networks". in the proceedings of the IEEE Workshop on Wireless Ad Hoc and Sensor Networks,2004. pp. 33- 38 .
[4] J. Ansari, X. Zhang, and P. Mähönen, "Multi-radio medium access control protocol for wireless sensor networks," in proceedings of the 5th International Conference on Embedded Networked Sensor Systems ACM, 2007, pp. 403-404.

[5] M. Kohvakka, T. Arpinen, M. Hännikäinen, and T. D. Hämäläinen, "Highperformance multi-radio WSN platform," in proceedings of the 2nd International Workshop on Multi-hop Ad Hoc networks: From Theory to Reality ACM, 2006.

[6] H. Steendam and M. Moeneclaey, "The Effect of Carrier Frequency Offsets on Downlink and Uplink MC-DS-CDMA," IEEE Journal on Selected Areas in Communications, vol. 19, no. 12, pp. 2528-2536, 2001.

[7] F. Qin, Y. Yang, and J. Mitchell, "Performance Increase Through the Use of Multiple Sub-carriers in WSN," in 7th ACM International Symposium on Mobility Management and Wireless Access, 2009, pp. 132-139.

[8] J. Deng, Y. S. Han, and Z. J. Haas, "Analyzing split channel medium access control schemes," IEEE Transactions on Wireless Communications, vol. 5, no. 5, p. 967-971, 2006.

[9] Y. S. Han, J. Deng, and Z. J. Haas, "Analyzing multi-channel medium access control schemes with ALOHA reservation," IEEE Transactions on Wireless Communications, vol. 5, no. 8, p. 2143-2152, 2006.

[10] L. Xinhua, C. Yu, J. W. Mark, and S. Xuemin, "A Renewal Theory Based Analytical Model for the Contention Access Period of IEEE 802.15.4 MAC," IEEE Transactions on Wireless Communications, vol. 7, no. 6, pp. 2340-2349, 2008.

[11] V. Istratescu, "Fixed Point Theory, an Introduction", Dordrecht, Holland, 1981.

[12] IEEE Std.802.15.4 Std, 2003.

[13] Texas Instruments, "CC2420 Datasheet," 2008. 